\title{
Evaluation of CYP17A1 and CYP1B1 polymorphisms in male breast cancer risk
}

\author{
Piera Rizzolo ${ }^{1, *}$, Valentina Silvestri ${ }^{1, *}$, Virginia Valentini ${ }^{1}$, Veronica Zelli ${ }^{1}$, Agostino Bucalo ${ }^{1}$, Ines Zanna ${ }^{2}$, \\ Simonetta Bianchi ${ }^{3}$, Maria Grazia Tibiletti ${ }^{4}$, Antonio Russo ${ }^{5}$, Liliana Varesco ${ }^{6}$, Gianluca Tedaldi ${ }^{7}$, Bernardo Bonanni ${ }^{8}$, \\ Jacopo Azzollini ${ }^{9}$, Siranoush Manoukiann ${ }^{9}$, Anna Coppa $^{10}$, Giuseppe Giannini ${ }^{1}$, Laura Cortesi ${ }^{11}$, Alessandra Viel ${ }^{12}$, \\ Marco Montagna ${ }^{13}$, Paolo Peterlongo ${ }^{14}$, Paolo Radice ${ }^{15}$, Domenico Palli ${ }^{2}$ and Laura Ottini ${ }^{1}$ \\ ${ }^{1}$ Department of Molecular Medicine, Sapienza University of Rome, Rome, Italy \\ ${ }^{2}$ Cancer Risk Factors and Lifestyle Epidemiology Unit, Institute for Cancer Research, Prevention and Clinical Network (ISPRO), Florence, Italy \\ ${ }^{3}$ Division of Pathological Anatomy, Department of Sciences of Health, University of Florence, Florence, Italy \\ ${ }^{4}$ Department of Pathology, ASST Settelaghi and Centro di Ricerca per lo Studio dei Tumori Eredo-Familiari, Università dell'Insubria, Varese, Italy \\ ${ }^{5}$ Section of Medical Oncology, Department of Surgical and Oncological and Oral Sciences, University of Palermo, Palermo, Italy \\ ${ }^{6}$ IRCCS Ospedale Policlinico San Martino, Genoa, Italy \\ ${ }^{7}$ Biosciences Laboratory, Istituto Scientifico Romagnolo per lo Studio e la Cura dei Tumori (IRST) IRCCS, Meldola, Italy \\ ${ }^{8}$ Division of Cancer Prevention and Genetics IEO, European Institute of Oncology IRCCS, Milan, Italy \\ ${ }^{9}$ Unit of Medical Genetics, Department of Medical Oncology and Hematology, Fondazione IRCCS Istituto Nazionale dei Tumori (INT), Milan, Italy \\ ${ }^{10}$ Department of Experimental Medicine, Sapienza University of Rome, Rome, Italy \\ ${ }^{11}$ Department of Oncology and Haematology, University of Modena and Reggio Emilia, Modena, Italy \\ ${ }^{12}$ Unit of Functional Onco-Genomics and Genetics, Centro di Riferimento Oncologico di Aviano (CRO), IRCCS, Aviano, Italy \\ ${ }^{13}$ Immunology and Molecular Oncology Unit, Veneto Institute of Oncology IOV - IRCCS, Padua, Italy \\ ${ }^{14}$ Genome Diagnostics Program, IFOM - The FIRC Institute of Molecular Oncology, Milan, Italy \\ ${ }^{15}$ Unit of Molecular Bases of Genetic Risk and Genetic Testing, Department of Research, Fondazione IRCCS Istituto Nazionale Tumori (INT), Milan, Italy
}

Correspondence should be addressed to L Ottini: laura.ottini@uniroma1.it

*(P Rizzolo and V Silvestri contributed equally to this work)

\begin{abstract}
Breast cancer in men is a rare and still poorly characterized disease. Inherited mutations in BRCA1, BRCA2 and PALB2 genes, as well as common polymorphisms, play a role in male breast cancer genetic predisposition. Male breast cancer is considered a hormonedependent tumor specifically related to hyperestrogenism. Polymorphisms in genes involved in estrogen biosynthesis and metabolism pathways, such as CYP17A1 and CYP1B1, have been associated with breast cancer risk. Here, we aimed to investigate the role of CYP17A1 and CYP1B1 polymorphisms in male breast cancer risk. A series of 597 male breast cancer cases and 1022 male controls, recruited within the Italian Multicenter Study on male breast cancer, was genotyped for CYP17A1 rs743572, CYP1B1 rs1056836 and rs1800440 polymorphisms by allelic discrimination real-time PCR with TaqMan probes. Associations with male breast cancer risk were estimated using logistic regression. No statistically significant associations between male breast cancer risk and the three analyzed polymorphisms emerged. Similar results were obtained also when BRCA1/2 mutational status was considered. No significant differences in the distribution of the genotypes according to estrogen receptor status emerged. In conclusion, our study, based on a large series of male breast cancer cases, is likely to exclude a relevant role of CYP17A1 and CYP1B1 polymorphisms in male breast cancer predisposition. Overall, these results add new data to the increasing evidence that polymorphisms in these genes may not be associated with breast cancer risk.
\end{abstract}
Key Words
- male breast cancer
- CYP17A1
- CYP1B1
- polymorphisms
- male breast cancer risk

https://ec.bioscientifica.com

https://doi.org/10.1530/EC-19-0225

(c) 2019 The authors Published by Bioscientifica Ltd

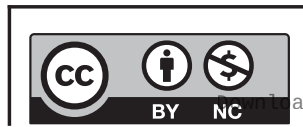

This work is licensed under a Creative Commons Attribution-NonCommercial 4.0 International License. ded from Bioscientifica.com at 04/26/2023 01:09:27AM
Endocrine Connections (2019) 8, 1224-1229 


\section{Introduction}

Male breast cancer (MBC) is a rare disease, representing about $1 \%$ of all breast cancers (BCs) and less than $1 \%$ of all cancers in men (1). Germline pathogenic variants in $\mathrm{BC}$ genes, particularly BRCA1, BRCA2 and PALB2 genes, increase the risk of developing $\operatorname{MBC}(2,3,4)$. Common polymorphisms may also contribute to $\mathrm{MBC}$ genetic predisposition and may have a modifying effect on $\mathrm{BC}$ risk for male BRCA1/2 mutation carriers, particularly through a polygenic inheritance model $(5,6,7)$.

MBC is recognized as a hormone-dependent malignancy and is widely considered as an estrogendriven disease, specifically related to hyperestrogenism $(1,8)$. Notably, most of male breast tumors are estrogen receptor (ER) positive (1). An increased level of circulating estradiol appears to be an important factor in the etiology of this disease and the mean total serum estradiol level is significantly increased in MBC patients compared with healthy males (8).

Polymorphisms in genes involved in estrogen biosynthesis and metabolism pathways, such as Cytochrome P450 family 17 subfamily A member 1 (CYP17A1) and Cytochrome P450 family 1 subfamily B member 1 (CYP1B1), may cause an increased risk of hormone-related cancers, such as $\mathrm{BC}$, by altering the expression of steroid hormones, including estrogens $(9,10,11)$

CYP17A1 is an enzyme essential for the biosynthesis of estrogens and androgens (12). A common polymorphism in the promoter of CYP17A1 (c.-34T>C; rs743572) has been associated with increased CYP17A1 expression, enhanced estrogen production and increased serum estradiol levels in post-menopausal women $(12,13)$. This polymorphism has been investigated in female BC (FBC) with controversial results $(12,13)$. To date, only two studies have analyzed a possible role of CYP17A1 rs743572 in MBC risk, with contrasting results $(14,15)$. A higher rs743572 CC genotype frequency among BRCA2 mutation carriers has been observed in a small MBC series, suggesting a possible effect of rs743572 polymorphism as a genetic modifier of $\mathrm{BC}$ risk (15).

CYP1B1 is a key enzyme in the initial catabolic step of estrogens metabolism. There are several common CYP1B1 polymorphisms known to affect its enzymatic efficiency, including CYP1B1 c.1294A >G (p.Leu432Val; rs1056836) and c.1358A $>$ G (p.Asn453Ser; rs1800440) (9). In particular, CYP1B1 rs1056836 has been associated with increased CYP1B1 catalytic activity, whereas CYP1B1 rs 1800440 has been associated with a decrease in protein expression due to degradation $(16,17)$. These two CYP1B1 polymorphisms have been widely investigated in female BC (FBC) with contrasting results $(18,19,20,21,22,23)$. To date, the role of CYP1B1 rs1056836 and rs1800440 polymorphisms in $\mathrm{MBC}$ risk has not been investigated.

In this study, we aimed to evaluate possible associations between CYP17A1 rs743572, CYP1B1 rs1056836 and rs1800440 polymorphisms and MBC risk, analyzing a large series of $\mathrm{MBC}$ cases characterized for $B R C A 1 / 2$ mutation status and ER status. Given that MBC is unencumbered by the many confounding factors that exist in FBC (for example, reproductive factors and high frequency), the investigation of CYP17A1 and CYP1B1 polymorphisms in men may be instrumental in giving insight into the role these polymorphisms play in $\mathrm{BC}$ and to provide information that may be inherent to the disease in both genders.

\section{Materials and methods}

\section{Study population}

A total of $597 \mathrm{MBC}$ cases and 1022 male Italian (Caucasian) controls were included in the present study. MBC cases, together with information about BRCA1/2 mutation status and the main clinical-pathologic characteristics, were recruited in the frame of the ongoing Italian Multicenter Study on MBC, as previously described (24). BRCA1 and $B R C A 2$ mutation analysis was first performed in the frame of genetic counseling programs at the center of origin for all $\mathrm{MBC}$ cases, then $B R C A 1 / 2$ mutation-negative cases were retested using next-generation sequencing (4). Overall, 89 out of $597 \mathrm{MBC}$ cases (15\%) were carriers of a pathogenic variant in BRCA1/2 genes (10 BRCA1; 79 BRCA2). Information on ER status was available for 448 MBCs; the majority of MBC cases were ER-positive tumors $(93.3 \%)$.

Of the 1022 male controls, 865 were male individuals without personal history of cancer (information about family history of cancer was not available), enrolled in hospital-based settings under research or clinical protocols at the same centers of MBC cases or blood donors $(5,24)$. Of the 865 control individuals 260 (30\%) were tested for $B R C A 1 / 2$ mutations and resulted negative. The additional 157 controls were male carriers of $B R C A 1 / 2$ mutations without personal history of cancer, recruited among hereditary breast and ovarian cancer families.

For each study participant, samples of blood or DNA from peripheral blood leukocytes were collected. DNA from

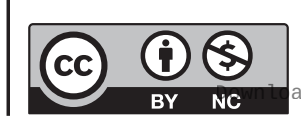


blood samples was extracted and quantified as previously described (5). The experimental protocol was approved by the Local Ethical Committee (Sapienza University of Rome, Number of Protocol 669/17). All procedures performed in the present study were in accordance with the ethical standards of the institutional research committee and with the 1964 Helsinki declaration and its later amendments. Informed consent was obtained from all participants included in the study.

\section{Genotyping}

$\mathrm{MBC}$ cases and controls were genotyped by allelic discrimination real-time PCR with TaqMan probes in ABI 7500 fast real-time PCR instrument (Life Technologies) at CYP17A1 rs743572, CYP1B1 rs1056836 and rs1800440, by commercially available assays (Life Technologies), according to the manufacturer's instruction. In each experiment, duplicates, positive (cases for which genotype was confirmed by Sanger sequencing) and negative (water) controls were included (24).

\section{Statistical analysis}

Statistical analyses were performed as previously described $(5,6,24)$. The genotype frequencies for each polymorphism were evaluated in both cases and controls. The association between polymorphisms and $\mathrm{MBC}$ risk was estimated using logistic regression after adjustment for age of participants, center of enrolment and ascertainment (population- or clinic-based) and was measured by the odds ratio (OR) and its corresponding 95\% confidence interval (CI). For each polymorphism, a specific model was used to evaluate separately the effect of the heterozygous and of the homozygous genotypes. We also evaluated MBC risk based on multiplicative co-dominant (per-allele) model. In each model, the common homozygote genotype in the control population was considered as the reference category. Considering a minor allele frequency of $20 \%$ (lower value in our control's series) and a dominant model, with a casecontrol ratio of 1:1.7 (597 cases and 1022 controls), we could identify an OR of 1.45 with a power of $90 \%$ and alpha $=0.05$.

Chi-square test was performed in a case-by-case analysis in order to evaluate the potential associations between genotypes and ER status. A $P$ value $<0.05$ was considered statistically significant. All analyses were performed using STATA version 13.1 statistical program.

\section{Results}

CYP17A1 rs743572, CYP1B1 rs1056836 and rs1800440 were genotyped in the whole series of 1619 individuals, including $597 \mathrm{MBC}$ cases and 1022 male controls.

The distribution of genotype frequencies of CYP17A1 rs743572, CYP1B1 rs1056836 and rs1800440 polymorphisms in $\mathrm{MBC}$ cases and controls and the risk estimates are summarized in Table 1.

No statistically significant associations between MBC risk and the three analyzed polymorphisms emerged by logistic regression models. The same results were obtained when all affected and unaffected BRCA1/2 mutation carriers were excluded from the analyses (data not shown).

The analysis was then restricted to male BRCA1/2 mutation carriers comparing the 89 BRCA1/2-related

Table 1 Distribution of 597 MBC cases and 1022 male population controls according to genotype frequencies of CYP17A1 rs743572, CYP1B1 rs1056836 and rs1800440 and MBC risk estimates.

\begin{tabular}{|c|c|c|c|c|c|}
\hline \multirow[b]{2}{*}{ Polymorphism } & \multirow[b]{2}{*}{ Genotype } & \multicolumn{2}{|c|}{ MBC cases $(n=597)$} & \multicolumn{2}{|c|}{ Male controls $(n=1022)$} \\
\hline & & $n$ & $\%$ & $n$ & $\%$ \\
\hline CYP17A1 с.-34T>C & $\mathrm{TT}$ & 190 & 31.8 & 347 & 33.9 \\
\hline \multirow[t]{3}{*}{ rs743572 } & $\mathrm{TC}$ & 301 & 50.4 & 489 & 47.9 \\
\hline & $\mathrm{CC}$ & 106 & 17.8 & 186 & 18.2 \\
\hline & Co-dominant & & & & \\
\hline CYP1B1 p.Leu432Val & $\mathrm{GG}$ & 231 & 38.7 & 385 & 37.7 \\
\hline \multirow[t]{3}{*}{ rs1056836 } & $\mathrm{GC}$ & 290 & 48.6 & 482 & 47.1 \\
\hline & $\mathrm{CC}$ & 76 & 12.7 & 155 & 15.2 \\
\hline & Co-dominant & & & & \\
\hline CYP1B1 p.Asn453Ser & $\mathrm{AA}$ & 365 & 61.2 & 654 & 64.0 \\
\hline \multirow[t]{3}{*}{ rs1800440 } & AG & 208 & 34.8 & 321 & 31.4 \\
\hline & GG & 24 & 4.0 & 47 & 4.6 \\
\hline & Co-dominant & & & & \\
\hline
\end{tabular}

\begin{tabular}{rrr} 
OR $(95 \% \mathrm{Cl})$ & & \\
\cline { 1 - 1 } $\begin{aligned} \text { Ref } \\
1.07(0.84-1.38)\end{aligned}$ & & 0.57 \\
$0.90(0.66-1.26)$ & & 0.57 \\
$0.97(0.83-1.14)$ & & 0.71 \\
Ref & & \\
$0.94(0.75-1.20)$ & & 0.66 \\
$0.79(0.56-1.12)$ & & 0.19 \\
$0.91(0.77-1.06)$ & & 0.23 \\
Ref & & \\
$1.13(0.89-1.43)$ & & 0.3 \\
$1.14(0.65-1.99)$ & & 0.64 \\
$1.11(0.91-1.34)$ & & 0.31 \\
\hline
\end{tabular}


MBC cases with the 157 unaffected male BRCA1/2 mutation carriers (Table 2). No statistically significant results emerged. The same results were obtained when only BRCA2 mutation carriers were considered (data not shown).

The distribution of the genotypes in MBC cases was further analyzed taking into account ER status. No significant differences in the distribution of the genotypes according to ER status emerged (Table 3).

\section{Discussion}

Estrogens play a relevant role in $\mathrm{MBC}$; thus, genetic polymorphisms of genes involved in estrogen metabolism may have an impact on MBC susceptibility. The possible role of CYP17A1 rs743572 polymorphism in MBC risk has been analyzed by two studies both examining a limited number (from 39 to 76) of MBC cases, more than 15 years ago $(14,15)$. One study reported a significant association of this polymorphism with increased MBC risk (14) and the other study failed to replicate these findings (15). Afterward, no other study aimed to investigate this polymorphism in MBC; nevertheless, CYP17A1 is consistently reported as a putative genetic risk factor for $\mathrm{MBC}$ in reviews on the field $(25,26,27,28,29)$. Our results, based on the largest collection reported to date of $\mathrm{MBC}$ patients undergoing CYP17A1 genotyping, are likely to exclude, with a good confidence, a relevant contribution of CYP17A1 rs743572 polymorphism in $\mathrm{MBC}$ risk. Overall, in line with the most recent findings in $\mathrm{FBC}(30,31,32)$, our data further support the lack of association between $C Y P 17 A 1$ polymorphism and overall BC risk.
To our knowledge, at present, there are no published data on the role of CYP1B1 polymorphisms in MBC; thus, our study is the first to investigate a possible association between the two most studied CYP1B1 common functional polymorphisms (CYP1B1 rs1056836 and rs1800440) and $\mathrm{BC}$ risk in men. Associations between these two CYP1B1 polymorphisms with $\mathrm{BC}$ risk in women has been reported in some populations $(18,19,20)$; however, more recent meta-analyses showed that there is no overall effect on FBC risk $(21,22,23)$. In line with these meta-analysis studies, our study provides no evidence that CYP1B1 rs1056836 and rs1800440 may contribute to MBC risk.

Notably, loci for these two candidate genes have never emerged in any of the Genome Wide Association Studies (GWAS) on both female and male BCs as reported in the GWAS Catalog (33), thus further proving for a marginal role, if any, in $\mathrm{BC}$ risk.

We also tested the hypothesis that CYP17A1 and $C Y P 1 B 1$ polymorphisms may modulate the risk of $\mathrm{BC}$ conferred by BRCA1/2 mutations in men, as previously suggested by a small study (15). Results from our study, comparing BRCA1/2-associated MBC cases with unaffected male $B R C A 1 / 2$ mutation carriers, showed no evidence for an association of CYP17A1 rs743572, CYP1B1 rs1056836 and rs1800440 polymorphisms with MBC risk, suggesting that these variants are not likely to modify $\mathrm{BC}$ risk in male $B R C A 1 / 2$ mutation carriers.

To the best of our knowledge, our series, collected in the frame of the ongoing Italian Multicenter Study on MBC (24), represents one of the largest MBC series ever assembled in a single country, for which BRCA1 and BRCA2 mutational status and clinical-pathologic data are available. In previous studies, this series allowed

Table 2 Distribution of $89 B R C A 1 / 2$-associated $M B C$ cases and 157 unaffected male BRCA1/2 mutation carriers according to genotype frequencies of CYP1A1 rs743572, CYP1B1 rs1056836 and rs1800440 and MBC risk estimates.

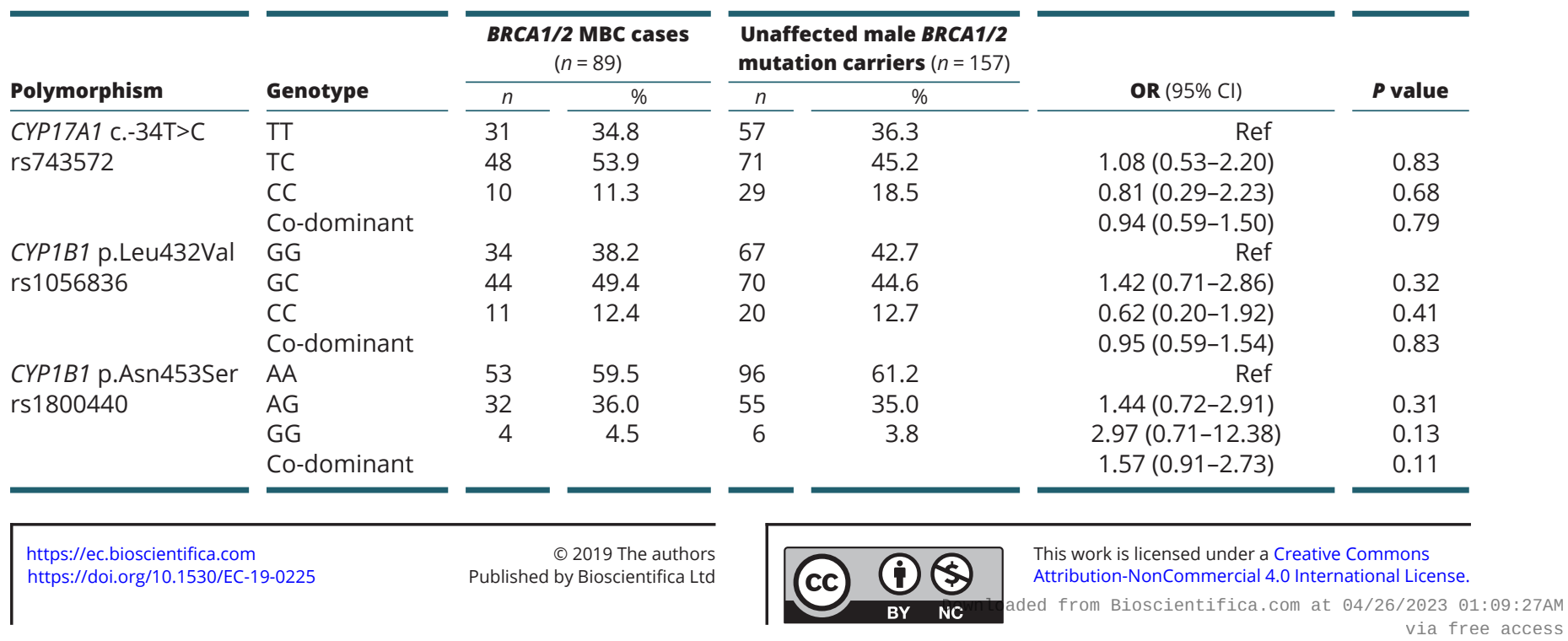


Table 3 Distribution of the three analyzed polymorphisms in the MBC case series according to ER status.

\begin{tabular}{|c|c|c|c|}
\hline \multirow[b]{2}{*}{ Polymorphism } & \multirow[b]{2}{*}{ Genotype } & \multicolumn{2}{|c|}{ ER-positive MBC cases $(n=418)$} \\
\hline & & $n$ & $\%$ \\
\hline CYP17A1 с. $-34 T>C$ & TT & 125 & 29.9 \\
\hline \multirow[t]{2}{*}{ rs743572 } & $\mathrm{TC}$ & 211 & 50.5 \\
\hline & $\mathrm{CC}$ & 82 & 19.6 \\
\hline CYP1B1 p.Leu432Val & GG & 161 & 38.5 \\
\hline \multirow[t]{2}{*}{ rs1056836 } & GC & 205 & 49.0 \\
\hline & $\mathrm{CC}$ & 52 & 12.5 \\
\hline CYP1B1 p.Asn453Ser & $\mathrm{AA}$ & 261 & 62.5 \\
\hline \multirow[t]{2}{*}{ rs1800440 } & AG & 141 & 33.7 \\
\hline & GG & 16 & 3.8 \\
\hline
\end{tabular}

\begin{tabular}{|c|c|c|}
\hline \multicolumn{2}{|c|}{ ER-negative MBC cases $(n=30)$} & \multirow[b]{2}{*}{ Chi $^{2}$ P-value } \\
\hline$n$ & $\%$ & \\
\hline 9 & 30.0 & 0.39 \\
\hline 18 & 60.0 & \\
\hline 3 & 10.0 & \\
\hline 8 & 26.7 & 0.31 \\
\hline 19 & 63.3 & \\
\hline 3 & 10.0 & \\
\hline 22 & 73.3 & 0.35 \\
\hline 8 & 26.7 & \\
\hline 0 & 0.0 & \\
\hline
\end{tabular}

for the identification of genetic polymorphisms as lowpenetrance susceptibility alleles in $\operatorname{MBC}(5,6,24)$. Thus, the power of the present study is adequate to detect risk effects similar to those previously reported $(14,18$, $19,20)$. On the other hand, the power of our study may be insufficient to identify smaller risk effects. However, smaller effects may be of little clinical relevance, unless included in the frame of a polygenic risk model (7). Large-scale collaborative studies are needed to investigate whether CYP17A1 and CYP1B1 genotypes may have a role in modulating anthropometric and epidemiologic risk factors in men.

In conclusion, our present findings, based on a large series of MBC cases and male controls, may exclude a relevant contribution of CYP17A1 and CYP1B1 polymorphisms in BC risk in men. Overall, these results add new data to the accumulating evidence that polymorphisms in these genes are not associated with $\mathrm{BC}$ risk in both genders.

\section{Declaration of interest}

The authors declare that there is no conflict of interest that could be perceived as prejudicing the impartiality of the research reported.

\section{Funding}

This work was supported by Associazione Italiana per la Ricerca sul Cancro (AIRC IG 21389) and Ricerca Scientifica di Ateneo La Sapienza to L O; Istituto Pasteur-Fondazione Cenci Bolognetti to G G; Italian Ministry of Education, Universities and Research - Dipartimenti di Eccellenza - L. 232/2016.

\section{Author contribution statement}

Pi R and V S drafted the manuscript, performed genotyping and statistical analyses and interpreted the results; $V V, V Z$ and $A B$ performed genotyping analysis; I Z, S B, M G T, A R, L V, G T, B B, J A, S M, A C, G G, L C, A V, M M, $P$ P,$~ P a ~ R$ and D P recruited samples and collected clinical-pathologic data; $\mathrm{L} O$ conceived, designed and coordinated the study. All authors reviewed, edited and approved the manuscript for publication.

\section{Acknowledgments}

The authors thank all the participants in this study and the institutions and their staff who supported the recruitment of patients and the collection of samples and data. The authors thank Dr Mario Falchetti for helpful discussion.

\section{References}

1 Ottini L. Male breast cancer: a rare disease that might uncover underlying pathways of breast cancer. Nature Reviews: Cancer 201414 643. (https://doi.org/10.1038/nrc3806)

2 Buffone A, Capalbo C, Ricevuto E, Sidoni T, Ottini L, Falchetti M, Cortesi E, Marchetti P, Scambia G, Tomao S, et al. Prevalence of BRCA1 and BRCA2 genomic rearrangements in a cohort of consecutive Italian breast and/or ovarian cancer families. Breast Cancer Research and Treatment 2007106 289-296. (https://doi. org/10.1007/s10549-007-9499-6)

3 Rizzolo P, Silvestri V, Tommasi S, Pinto R, Danza K, Falchetti M, Gulino M, Frati P \& Ottini L. Male breast cancer: genetics, epigenetics, and ethical aspects. Annals of Oncology 201324 (Supplement 8) viii75-viii82. (https://doi.org/10.1093/annonc/ mdt316)

4 Rizzolo P, Zelli V, Silvestri V, Valentini V, Zanna I, Bianchi S, Masala G, Spinelli AM, Tibiletti MG, Russo A, et al. Insight into genetic susceptibility to male breast cancer by multigene panel testing: results from a multicenter study in Italy. International Journal of Cancer 2019145 390-400. (https://doi.org/10.1002/ijc.32106) 5 Ottini L, Silvestri V, Saieva C, Rizzolo P, Zanna I, Falchetti M, Masala G, Navazio AS, Graziano V, Bianchi S, et al. Association of low-penetrance alleles with male breast cancer risk and clinicopathological characteristics: results from a multicenter study in Italy. Breast Cancer Research and Treatment 2013138 861-868. (https://doi.org/10.1007/s10549-013-2459-4)

6 Silvestri V, Rizzolo P, Scarnò M, Chillemi G, Navazio AS, Valentini V, Zelli V, Zanna I, Saieva C, Masala G, et al. Novel and known genetic variants for male breast cancer risk at 8q24.21, 9p21.3, 11q13.3 and 14q24.1: results from a multicenter study in Italy. European Journal of Cancer 201551 2289-2295. (https://doi.org/10.1016/j. ejca.2015.07.020)

7 Lecarpentier J, Silvestri V, Kuchenbaecker KB, Barrowdale D, Dennis J, McGuffog L, Soucy P, Leslie G, Rizzolo P, Navazio AS, et al. Prediction of breast and prostate cancer risks in male BRCA1 and BRCA2 mutation carriers using polygenic risk scores. Journal of Clinical Oncology 201735 2240-2250. (https://doi.org/10.1200/ JCO.2016.69.4935)

8 Brinton LA, Key TJ, Kolonel LN, Michels KB, Sesso HD, Ursin G, Van Den Eeden SK, Wood SN, Falk RT, Parisi D, et al. Prediagnostic sex steroid hormones in relation to male breast cancer risk. Journal

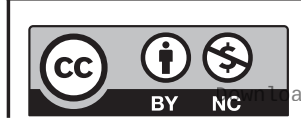

This work is licensed under a Creative Commons Attribution-NonCommercial 4.0 International License. ded from Bioscientifica.com at 04/26/2023 01:09:27 AM 
of Clinical Oncology 201533 2041-2050. (https://doi.org/10.1200/ JCO.2014.59.1602)

9 Gajjar K, Martin-Hirsch PL \& Martin FL. CYP1B1 and hormoneinduced cancer. Cancer Letters 2012324 13-30. (https://doi. org/10.1016/j.canlet.2012.04.021)

10 Blackburn HL, Ellsworth DL, Shriver CD \& Ellsworth RE. Role of cytochrome P450 genes in breast cancer etiology and treatment: effects on estrogen biosynthesis, metabolism, and response to endocrine therapy. Cancer Causes and Control 201526 319-332. (https://doi.org/10.1007/s10552-014-0519-7)

11 Go RE, Hwang KA \& Choi KC. Cytochrome P450 1 family and cancers. Journal of Steroid Biochemistry and Molecular Biology 2015147 24-30. (https://doi.org/10.1016/j.jsbmb.2014.11.003)

12 Chen Y \& Pei J. Factors influencing the association between CYP17 T34C polymorphism and the risk of breast cancer: meta-regression and subgroup analysis. Breast Cancer Research and Treatment 2010122 471-481. (https://doi.org/10.1007/s10549-009-0690-9)

13 Tworoger SS, Chubak J, Aiello EJ, Ulrich CM, Atkinson C, Potter JD, Yasui Y, Stapleton PL, Lampe JW, Farin FM, et al. Association of CYP17, CYP19, CYP1B1, and COMT polymorphisms with serum and urinary sex hormone concentrations in postmenopausal women. Cancer Epidemiology, Biomarkers and Prevention 200413 94-101. (https://doi.org/10.1158/1055-9965.EPI-03-0026)

14 Young IE, Kurian KM, Annink C, Kunkler IH, Anderson VA, Cohen BB, Hooper ML, Wyllie AH \& Steel CM. A polymorphism in the CYP17 gene is associated with male breast cancer. British Journal of Cancer 199981 141-143. (https://doi.org/10.1038/sj.bjc.6690663)

15 Gudmundsdottir K, Thorlacius S, Jonasson JG, Sigfusson BF, Tryggvadottir L \& Eyfjord JE. CYP17 promoter polymorphism and breast cancer risk in males and females in relation to BRCA2 status. British Journal of Cancer 200388 933-936. (https://doi.org/10.1038/ sj.bjc.6600839)

16 Bandiera S, Weidlich S, Harth V, Broede P, Ko Y \& Friedberg T. Proteasomal degradation of human CYP1B1: effect of the Asn453Ser polymorphism on the post-translational regulation of CYP1B1 expression. Molecular Pharmacology 200567 435-443. (https://doi. org/10.1124/mol.104.006056)

17 Landi MT, Bergen AW, Baccarelli A, Patterson DG Jr, Grassman J, TerMinassian M, Mocarelli P, Caporaso N, Masten SA, Pesatori AC, et al. CYP1A1 and CYP1B1 genotypes, haplotypes, and TCDD-induced gene expression in subjects from Seveso, Italy. Toxicology $2005 \mathbf{2 0 7}$ 191-202. (https://doi.org/10.1016/j.tox.2004.08.021)

18 Li D, Walcott FL, Chang P, Zhang W, Zhu J, Petrulis E, Singletary SE, Sahin AA \& Bondy ML. Genetic and environmental determinants on tissue response to in vitro carcinogen exposure and risk of breast cancer. Cancer Research 200262 4566-4570.

19 Gaudet MM, Chanock S, Lissowska J, Berndt SI, Yang XR, Peplonska B, Brinton LA, Welch R, Yeager M, Bardin-Mikolajczak A, et al. Genetic variation of cytochrome P450 1B1 (CYP1B1) and risk of breast cancer among Polish women. Pharmacogenetics and Genomics 200616 547-553. (https://doi.org/10.1097/01. fpc.0000215067.29342.6f)

20 Matyjasik J, Cybulski C, Masojé B, Jakubowska A, SerranoFernandez P, Górski B, Debniak T, Huzarski T, Byrski T, Gronwald J, et al. CYP1B1 and predisposition to breast cancer in Poland. Breast
Cancer Research and Treatment 2007106 383-388. (https://doi. org/10.1007/s10549-007-9500-4)

21 Economopoulos KP \& Sergentanis TN. Three polymorphisms in cytochrome P450 1B1 (CYP1B1) gene and breast cancer risk: a metaanalysis. Breast Cancer Research and Treatment 2010122 545-551. (https://doi.org/10.1007/s10549-009-0728-z)

22 Yao L, Fang F, Wu Q, Zhong Y \& Yu L. No association between CYP1B1 Val432Leu polymorphism and breast cancer risk: a metaanalysis involving 40,303 subjects. Breast Cancer Research and Treatment 2010122 237-242. (https://doi.org/10.1007/s10549-0090689-2)

23 Liu JY, Yang Y, Liu ZZ, Xie JJ, Du YP \& Wang W. Association between the CYP1B1 polymorphisms and risk of cancer: a meta-analysis. Molecular Genetics and Genomics 2015290 739-765. (https://doi. org/10.1007/s00438-014-0946-x)

24 Rizzolo P, Silvestri V, Bucalo A, Zelli V, Valentini V, Catucci I, Zanna I, Masala G, Bianchi S, Spinelli AM, et al. Contribution of MUTYH variants to male breast cancer risk: results from a multicenter study in Italy. Frontiers in Oncology 20188 583. (https://doi.org/10.3389/ fonc.2018.00583)

25 Ruddy KJ \& Winer EP. Male breast cancer: risk factors, biology, diagnosis, treatment, and survivorship. Annals of Oncology 201324 1434-1443. (https://doi.org/10.1093/annonc/mdt025)

26 Ferzoco RM \& Ruddy KJ. The epidemiology of male breast cancer. Current Oncology Reports 201618 1. (https://doi.org/10.1007/s11912015-0487-4)

27 Abdelwahab Yousef AJ. Male breast cancer: epidemiology and risk factors. Seminars in Oncology 201744 267-272. (https://doi. org/10.1053/j.seminoncol.2017.11.002)

28 Giordano SH. Breast cancer in men. New England Journal of Medicine 2018378 2311-2320. (https://doi.org/10.1056/NEJMra1707939)

29 Gucalp A, Traina TA, Eisner JR, Parker JS, Selitsky SR, Park BH, Elias AD, Baskin-Bey ES \& Cardoso F. Male breast cancer: a disease distinct from female breast cancer. Breast Cancer Research and Treatment 2019173 37-48. (https://doi.org/10.1007/s10549-0184921-9)

30 Mao C, Wang XW, He BF, Qiu LX, Liao RY, Luo RC \& Chen Q. Lack of association between CYP17 MspA1 polymorphism and breast cancer risk: a meta-analysis of 22,090 cases and 28,498 controls. Breast Cancer Research and Treatment 2010122 259-265. (https://doi. org/10.1007/s10549-009-0695-4)

31 Yao L, Fang F, Wu Q, Yang Z, Zhong Y \& Yu L. No association between CYP17 T-34C polymorphism and breast cancer risk: a meta-analysis involving 58,814 subjects. Breast Cancer Research and Treatment 2010122 221-227. (https://doi.org/10.1007/s10549-0090679-4)

32 Sun J, Zhang H, Gao M, Tang Z, Guo D, Zhang X, Wang Z, Li R, Liu Y, Sun W, et al. Association between CYP17 T-34C rs743572 and breast cancer risk. Oncotarget 20189 4200-4213. (https://doi.org/10.18632/ oncotarget.23688)

33 Buniello A, MacArthur JAL, Cerezo M, Harris LW, Hayhurst J, Malangone C, McMahon A, Morales J, Mountjoy E, Sollis E, et al. The NHGRI-EBI GWAS catalog of published genome-wide association studies, targeted arrays and summary statistics 2019. Nucleic Acids Research 201947 D1005-D1012. (https://doi.org/10.1093/nar/gky1120)

Received in final form 4 July 2019

Accepted 23 July 2019

Accepted Preprint published online 23 July 2019 https://ec.bioscientifica.com https://doi.org/10.1530/EC-19-0225 (c) 2019 The authors Published by Bioscientifica Ltd

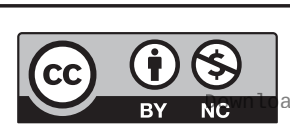

This work is licensed under a Creative Commons Attribution-NonCommercial 4.0 International License. ded from Bioscientifica.com at 04/26/2023 01:09:27AM 